Revisión

\title{
Sexualidad y patogenicidad en Aspergillus fumigatus: ¿existe alguna relación?
}

\author{
Sergio Álvarez-Pérez*, José Luis Blanco, Patricia Alba y Marta E. García \\ Departamento de Sanidad Animal, Facultad de Veterinaria, Universidad Complutense de Madrid, Madrid, España
}

I N F O R M A C IÓN DEL ARTÍCULO

Historia del artículo:

Recibido el 11 de agosto de 2009

Aceptado el 30 de noviembre de 2009

On-line el 18 de febrero de 2010

Palabras clave:

Aspergillus fumigatus

Locus MAT

Reproducción sexual

Patogenicidad

\section{R E S U M E N}

Antecedentes: Aspergillus fumigatus, al igual que muchas otras especies fúngicas de importancia clínica, ha sido tradicionalmente considerado un organismo asexual. Sin embargo, en los últimos años, diversas líneas de evidencia cuestionaron tal asunción, sugiriendo que el estado sexual de A. fumigatus podría permanecer aún por descubrir. Estas investigaciones han conducido finalmente al reciente descubrimiento de una fase teleomorfa de A. fumigatus, a la cual se le ha denominado Neosartorya fumigata.

Objetivos: Revisar los principales hallazgos realizados sobre la sexualidad de A. fumigatus y discutir las posibles implicaciones de tales hallazgos sobre su patogenicidad.

Métodos: Se realizó una búsqueda bibliográfica de los principales trabajos que abordan el estudio de la sexualidad de algunos patógenos fúngicos y, en especial, de A. fumigatus. Además, se incorporaron a la discusión algunos datos proporcionados por nuestra reciente experiencia investigadora sobre el tema Resultados: La existencia de un teleomorfo de A. fumigatus podría tener grandes repercusiones desde un punto de vista clínico, ya que como resultado de la reproducción sexual se podría originar una descendencia con mayor virulencia y/o resistencia a los agentes antifúngicos. En este sentido, los resultados de nuestras propias investigaciones sugieren la existencia de una asociación significativa entre el tipo sexual MAT1-1 y la invasividad de los aislamientos de A. fumigatus.

Conclusiones: El estudio de la reproducción sexual de los patógenos fúngicos y su posible relación con la virulencia seguirá siendo un tema de interés en los próximos años, no sólo por su interés básico sino también por sus posibles repercusiones clínicas.

(c) 2009 Revista Iberoamericana de Micología. Publicado por Elsevier España, S.L. Todos los derechos reservados.

\section{Sexuality and pathogenicity in Aspergillus fumigatus: Is there any relationship?}

\section{A B S T R A C T}

Background: Aspergillus fumigatus, like many other fungal species of clinical relevance, has been traditionally regarded as an asexual organism. However, in last few years several pieces of evidence question this such assumption, suggesting that the sexual state of $A$. fumigatus may still be undiscovered. These investigations have finally led to the recent discovery of a teleomorph stage of $A$. fumigatus, which has been named Neosartorya fumigata.

Aims: To review the most important findings on A. fumigatus sexuality and discuss the possible implications of such findings on its pathogenicity.

Methods: A bibliographic search was performed to find the main works that study the sexuality of fungal pathogens and, especially, of $A$. fumigatus. Moreover, data from our recent investigations in this field were also introduced to the discussion.

Results: The existence of a teleomorph for A. fumigatus could have significant clinical repercussions, as sexual reproduction might produce offspring with increased virulence and/or resistance to antifungal agents. In this sense, the results of our investigations suggest the existence of an association between the MAT1-1 mating type and the invasiveness of A. fumigatus isolates.

Conclusions: The study of the sexual reproduction of the fungal pathogens and its possible relationship with virulence will continue to be a topic of interest during the next years, not only because of its basic interest, but also for the possible clinical repercussions.

(c) 2009 Revista Iberoamericana de Micología. Published by Elsevier España, S.L. All rights reserved.

\footnotetext{
* Autor para correspondencia.

E-mail address: saperez@vet.ucm.es (S. Álvarez-Pérez).
}

El estudio de los estados sexuales o teleomorfos es la base de la taxonomía y nomenclatura fúngica ${ }^{21,24}$. Sin embargo, se estima que la quinta parte de las especies de hongos descritas sólo son conocidas por sus estructuras asexuales ${ }^{43}$. 
Hasta hace poco tiempo, Aspergillus fumigatus formaba parte de este grupo de hongos considerados exclusivamente asexuales. Sin embargo, diversos hallazgos realizados en los últimos años llevaron a cuestionar la supuesta asexualidad de este patógeno oportunista, sugiriendo que podría existir algún estadio sexual aún por descubrir o algún otro mecanismo para promover la recombinación sexual. El reciente descubrimiento de una fase sexual para esta especie anamorfa, a la cual se ha denominado Neosartorya fumigata O'Gorman, Fuller \& Dyer sp. nov. ${ }^{37}$, viene a resolver estas dudas, y confirma la pertenencia de A. fumigatus al Phylum Ascomycota.

El descubrimiento del ciclo sexual de A. fumigatus, y otros hongos de importancia médica y veterinaria, ha generado cierta expectación entre los micólogos, de modo que existe en la actualidad un gran interés en elucidar si existe alguna relación, directa o indirecta, entre la reproducción sexual y la patogenicidad fúngica. En este sentido, resulta especialmente inquietante la posibilidad de que, como resultado de los procesos de reproducción sexual, puedan aparecer nuevas cepas de mayor virulencia y/o resistencia a los antifúngicos.

En este artículo se presentan los principales descubrimientos que han llevado a la descripción del ciclo sexual de A. fumigatus. Además, se analiza la posible relación entre la reproducción sexual y la patogenicidad de este hongo.

\section{El ciclo sexual de Aspergillus fumigatus}

Aunque hasta hace poco tiempo no se había observado la ocurrencia de reproducción sexual en A. fumigatus, diversas líneas de evidencia habían llevado a sugerir la posible existencia de una fase teleomorfa de esta especie, que se ubicaría dentro del Phylum Ascomycota. En la tabla 1 se presentan algunas de estas evidencias, así como la bibliografía de consulta recomendada.

Pese a la importancia de todos estos hallazgos preliminares, debe tenerse en cuenta que se referían tan solo a la «potencialidad» de A. fumigatus para llevar a cabo la reproducción sexual y no a una situación real comprobada experimentalmente. De hecho, para explicar la aparente asexualidad de $A$. fumigatus, se llegó a contemplar la posibilidad de que esta especie hubiese perdido en el curso de su evolución parte de la maquinaria genética necesaria para la reproducción sexual ${ }^{12,44}$.

La demostración definitiva de que A. fumigatus mantiene su potencial sexual ha sido llevada a cabo recientemente por O'Gorman et $\mathrm{al}^{37}$, quienes han logrado reproducir en el labora- torio el ciclo sexual completo de esta especie, llegando a observar la producción de cleistotecios y ascosporas. En base a sus relaciones filogenéticas y a la morfología de sus estructuras sexuales, el teleomorfo de A. fumigatus ha sido asignado al género Neosartorya (Phylum Ascomycota, Orden eurotiales, Familia Trichocomaceae ${ }^{27}$ ), pasándose a denominar $N$. fumigata ${ }^{37}$. No obstante, hay quien considera que para evitar confusiones nomenclaturales debería prevalecer la denominación de $A$. fumigatus para referirse tanto a la fase asexual como al teleomorfo de este hongo, tal y como viene haciéndose con otras especies del género Aspergillus ${ }^{22}$.

El trabajo de O'Gorman et $\mathrm{al}^{37}$ proporciona además algunas claves para intentar explicar por qué no se había descubierto antes el ciclo sexual de A. fumigatus.

En primer lugar, el ciclo sexual de A. fumigatus parece requerir un tiempo más prolongado para ser completado del que necesitan la mayoría de especies de Aspergillus. De hecho, la maduración de los cleistotecios y la producción de ascosporas viables lleva un tiempo de incubación de hasta seis meses, si bien tras 6 u 8 semanas ya empiezan a aparecer agregaciones de hifas que recuerdan a cleistotecios inmaduros.

Por otro lado, podría suceder que los parámetros ambientales necesarios para favorecer el ciclo sexual de $A$. fumigatus se presenten tan solo de manera ocasional en la naturaleza. También podría ocurrir que dicho ciclo sexual esté confinado a determinados sustratos que no han sido monitorizados de forma sistemática. A este respecto, cabe destacar que O'Gorman et $\mathrm{al}^{37}$ solo observaron la producción de cleistotecios en los cruces realizados en agar harina de avena e incubados a $30^{\circ} \mathrm{C}$ en condiciones de oscuridad, pero no en los cruces realizados en agar extracto de malta, medio completo de Aspergillus o agar Czapek Dox, ni a ninguna de las otras 2 temperaturas probadas $\left(15^{\circ} \mathrm{C}\right.$ y $\left.45^{\circ} \mathrm{C}\right)$.

Finalmente, también podría ocurrir que la recombinación sexual en $A$. fumigatus esté restringida a pequeños grupos de aislamientos fértiles. En este sentido, los propios autores sugieren que la muestra estudiada en su trabajo podría representar tan solo un subgrupo infrecuente de aislamientos, todos ellos de origen ambiental y obtenidos en distintos puntos de Dublín (Irlanda), que habrían mantenido su fertilidad ${ }^{37}$. Esta idea va en la línea de la hipótesis que sugiere que las especies no deben ser vistas simplemente como sexuales o asexuales, sino como un conjunto de aislamientos individuales que se sitúan dentro de un continuum de fertilidad sexual ${ }^{12}$. De este modo, dentro de una

Tabla 1

Principales evidencias que llevaron a sugerir en el pasado la existencia de un ciclo sexual en Aspergillus fumigatus

\begin{tabular}{|c|c|}
\hline Ámbito & Evidencias \\
\hline Relaciones taxonómicas & $\begin{array}{l}\text { A. fumigatus está relacionado filogenéticamente con otras especies fúngicas capaces de reproducirse } \\
\text { sexualmente }\end{array}$ \\
\hline Estudios de genética de poblaciones & $\begin{array}{l}\text { La gran diversidad de genotipos de } A \text {. fumigatus en el ambiente contrasta con la supuesta asexualidad } \\
\text { atribuida tradicionalmente a esta especie }{ }^{11,12,30}\end{array}$ \\
\hline Estudios genómicos & $\begin{array}{l}\text { En el genoma de } A \text {. fumigatus se presentan los genes que determinan la compatibilidad sexual en hongos } \\
\text { ascomicetos, así como otras secuencias implicadas en las rutas de producción de feromonas y respuesta } \\
\text { a las mismas, el desarrollo del cuerpo fructífero y el proceso de la meiosis }{ }^{12,13,16,20,40,44}\end{array}$ \\
\hline Distribución de tipos sexuales & $\begin{array}{l}\text { Algunos autores han observado una relación de tipos sexuales de } A \text {. fumigatus de aproximadamente } 1: 1 \text {, } \\
\text { tanto en muestras clínicas como en muestras ambientales }{ }^{37,39,42} \text {. Tal distribución de los tipos sexuales } \\
\text { compatibles sugiere que la reproducción sexual está teniendo lugar de manera frecuente en las } \\
\text { poblaciones del hongo analizadas }{ }^{10} \text {. No obstante, otros autores han encontrado una mayor prevalencia } \\
\text { del tipo MAT } 1-2^{4}\end{array}$ \\
\hline Expresión de genes relacionados con el ciclo sexual & $\begin{array}{l}\text { Se ha detectado la expresión de los genes de tipo sexual (MAT1-1 y MAT1-2), de un gen que codifica para } \\
\text { un precursor de una feromona ( } p p g A) \text { y de los genes que codifican receptores de feromonas (preA y } \\
\text { preB), durante el cultivo en el laboratorio de cepas de } A \text {. fumigatus }{ }^{39}\end{array}$ \\
\hline
\end{tabular}


misma especie, los diferentes aislamientos podrían manifestar distinta capacidad para llevar a cabo la reproducción sexual ${ }^{12}$.

En cualquier caso, la presencia de un ciclo sexual en $A$. fumigatus tiene importantes implicaciones clínicas, ya que los cruces entre cepas compatibles del hongo podrían dar lugar a descendientes que presenten una mayor virulencia o una mayor resistencia a los agentes antifúngicos ${ }^{12,18,37}$. Además, la posibilidad de inducir en el laboratorio la ocurrencia de un ciclo sexual completo en A. fumigatus podría facilitar de manera considerable el análisis de la herencia de muchos de los caracteres relacionados con la patogenicidad de esta especie.

\section{Relación de la reproducción sexual con la patogenicidad}

\section{Reproducción sexual y estrategias de virulencia}

El papel que juega la reproducción sexual en la evolución de las especies de hongos patógenos es aún objeto de debate. Por un lado, la virulencia de la mayoría de los patógenos fúngicos es considerada un carácter multifactorial, por lo que la distribución al azar de los cromosomas y la recombinación que tienen lugar durante la meiosis podría romper combinaciones favorables de genes necesarios para la virulencia ${ }^{25}$. Sin embargo, también se puede utilizar el argumento opuesto, que defiende que esos mismos procesos pueden contribuir a generar descendencia que presente una mayor virulencia ${ }^{25}$.

Además de los aspectos relacionados con la generación de variabilidad genética, la reproducción sexual tiene otras ventajas e inconvenientes para el organismo que la lleva a cabo, algunas de las cuales se presentan en la tabla 2. Teniendo en cuenta este balance de ventajas e inconvenientes atribuibles a la reproducción sexual, cabe preguntarse por qué $A$. fumigatus y muchos otros patógenos fúngicos limitan al máximo la aparición de sus estados teleomorfos. Aunque todavía no existe una respuesta clara a esta cuestión, algunos autores sugieren que la limitación de la reproducción sexual podría ser una estrategia de virulencia ${ }^{23}$. Según esta hipótesis, muchos agentes patógenos se reproducen asexualmente para generar poblaciones clonales bien adaptadas a su hospedador y a sus nichos ambientales, pero, al mismo tiempo, mantienen la capacidad para reproducirse sexualmente en respuesta a cambios extremos en las condiciones ambientales o dentro del hospedador ${ }^{15,23,35}$. De este modo, la reproducción sexual permitiría a estos organismos expandir su rango ambiental, infectar nuevos hospedadores, evadir el sistema inmune o hacer frente a la terapia antimicrobiana ${ }^{15}$.

\section{Evidencias experimentales de la relación entre la sexualidad y la patogenicidad fúngica}

Aunque son muchos los autores que han discutido las posibles implicaciones de la reproducción sexual sobre la patogenicidad fúngica, actualmente existen pocas evidencias experimentales que sirvan para confirmar o refutar la mayoría de las hipótesis planteadas.

Entre las especies fúngicas de importancia clínica para las que se dispone de un mayor conocimiento sobre el ciclo sexual y su posible relación con la patogenicidad destaca Cryptococcus neoformans. Este conocimiento procede de los resultados obtenidos en diferentes estudios experimentales, algunos de los cuales se resumen en la tabla 3 .

A diferencia de $C$. neoformans, en el caso de A. fumigatus todavía no se han llevado a cabo trabajos experimentales que permitan relacionar la ocurrencia de un ciclo sexual con la patogenicidad. Tampoco se tienen datos sobre las posibles diferencias entre los tipos MAT1-1 y MAT1-2, en cuanto a su patogenicidad o resistencia a los agentes antifúngicos. De hecho, hasta hace poco solo se había estudiado la distribución de tipos sexuales en aislamientos ambientales y clínicos, observándose una relación $1: 1^{37,39,42}$ o una prevalencia algo superior del tipo MAT 1-2 .

Esta distribución de tipos sexuales, mucho menos desequilibrada que la observada en otros patógenos fúngicos, no parece sugerir una diferencia entre los tipos sexuales de A. fumigatus en cuanto a su virulencia. Sin embargo, los resultados de un trabajo llevado a cabo recientemente en nuestro laboratorio vienen a cuestionar esta afirmación. Concretamente, tras caracterizar 102 cepas de A. fumigatus de diferente origen (tanto ambientales, como procedentes de pacientes colonizados, con aspergiloma o con aspergilosis invasiva) pudimos constatar que el tipo sexual MAT1-1 no solo fue más prevalente entre los aislamientos clínicos de nuestra muestra, sino que, además, mostró una asociación estadísticamente signifi-

Tabla 2

Principales ventajas e inconvenientes asociados a la reproducción sexual*

\begin{tabular}{|c|c|}
\hline Ventajas & Inconvenientes \\
\hline Posible generación de nuevas combinaciones de caracteres favorables $3,12,26,46$ & Posibilidad de que se rompan combinaciones de genes favorables $5,12,38,46$ \\
\hline Mayor capacidad de adaptación a ambientes cambiantes ${ }^{3,26}$ & Grandes requerimientos energéticos ${ }^{12,38}$ \\
\hline Mayor posibilidad de eliminación de mutaciones deletéreas ${ }^{26,33,46}$ & Mayor lentitud que en el caso de la reproducción sexual ${ }^{38}$ \\
\hline $\begin{array}{l}\text { Reparación de posibles daños en la estructura del ADN durante el proceso de } \\
\text { recombinación sexual }\end{array}$ & \\
\hline
\end{tabular}

* Estas ventajas e inconvenientes están expresadas desde el punto de vista del organismo que lleva a cabo la reproducción sexual.

Tabla 3

Evidencias experimentales que sugieren la existencia de una estrecha relación entre sexualidad y patogenicidad en Cryptococcus neoformans

\begin{tabular}{|c|c|}
\hline Evidencias experimentales & Referencias \\
\hline El tipo sexual $\alpha$ de $C$. neoformans es mucho más prevalente que el tipo $a$, tanto en muestras clínicas como ambientales & 28,32 \\
\hline $\begin{array}{l}\text { El tipo } \alpha \text { de } C \text {. neoformans var. neoformans (serotipo D) produce en ratones una mayor mortalidad y de manera más temprana que } \\
\text { el tipo } a\end{array}$ & 29 \\
\hline $\begin{array}{l}\text { En experimentos de coinfección en ratones, el tipo sexual } \alpha \text { de } C \text {. neoformans var. grubii (serotipo A) muestra una mayor predilección } \\
\text { por diseminarse al sistema nervioso central que el tipo } a\end{array}$ & 34 \\
\hline $\begin{array}{l}\text { En células de } C \text {. neoformans serotipo A internalizadas por macrófagos murinos mantenidos en cultivos celulares se incrementa la } \\
\text { expresión de casi todos los genes del locus MAT }\end{array}$ & 14 \\
\hline
\end{tabular}


cativa con el origen invasivo de los aislamientos ${ }^{1}$. Por otro lado, también observamos que el tipo sexual MAT1-1 parece estar relacionado con la actividad elastasa, siendo más prevalente entre los aislamientos que presentan una mayor capacidad para degradar este sustrato (índice de actividad elastasa $\geq 1$ ) ${ }^{1}$. Esta última observación debe ponerse en el contexto de otros trabajos llevados a cabo anteriormente por nuestro grupo de investigación, que sugieren la importancia de la actividad elastasa como posible factor implicado en la patogenicidad de A. fumigatus ${ }^{8,17}$.

Pese al interés de nuestros resultados experimentales, debe reconocerse que la asociación existente entre el tipo sexual de A. fumigatus y sus posibles factores de virulencia es aún especulativa, pues no se ha encontrado hasta ahora prueba alguna de que el locus MAT esté ligado a algún otro locus relacionado con la patogenicidad de esta especie. Además, la posible asociación entre el tipo sexual y la patogenicidad de A. fumigatus debería ser confirmada en modelos animales mediante experiencias de infección simple y coinfección. Esta aproximación experimental, que permitiría identificar cualquier posible ventaja de uno de los 2 tipos sexuales de A. fumigatus en la colonización e infección del hospedador, requeriría la construcción de cepas isogénicas que se diferencien tan solo en el locus MAT.

Por otro lado, resulta interesante el hecho de que diferentes aislamientos de $A$. fumigatus procedentes de un mismo paciente puedan pertenecer a diferente tipo sexual, tal y como han descrito Paoletti et $\mathrm{al}^{39} \mathrm{y}$ hemos confirmado recientemente en nuestro laboratorio ${ }^{2}$. Esta observación puede considerarse indicativa de la ocurrencia de infecciones policlonales por este hongo oportunista, ya que los aislamientos de diferente tipo sexual han de ser necesariamente distintos, independientemente de los resultados de las técnicas de tipificación molecular.

La coexistencia de cepas de A. fumigatus sexualmente compatibles en el interior de un hospedador también puede llevarnos a plantear cuestiones tales como la posibilidad de que los 2 tipos sexuales de A. fumigatus tengan predilección por diseminarse a distintos tejidos y órganos, o, incluso, que pudieran llegar a aparearse dentro del hospedador. Esta última posibilidad, en caso de demostrarse, sería ciertamente preocupante, ya que la aparición de nuevas variantes genéticas del hongo durante el curso de una colonización o infección podría llegar a comprometer la eficacia de los tratamientos frente a la aspergilosis. Sin embargo, solo se ha observado hasta el momento la reproducción sexual de A. fumigatus a $30^{\circ} \mathrm{C}^{37}$, por lo que, al menos en principio, parece poco probable que las cepas compatibles puedan aparearse en los órganos y tejidos internos de aves y mamíferos. Además, todavía no se ha demostrado la expresión de los genes relacionados con el ciclo sexual de A. fumigatus bajo condiciones diferentes al cultivo en el laboratorio, como puede ser la infección experimental de cultivos celulares o en modelos animales de aspergilosis.

Finalmente, debe tenerse en cuenta que el carácter multifactorial de la patogenicidad de $A$. fumigatus $7,9,30,31,41$ puede complicar el análisis de la relación entre el tipo sexual y la virulencia. Por ejemplo, podría ocurrir que el tipo sexual esté relacionado con ciertos factores de patogenicidad pero no con otros, de manera que 2 cepas de distinto tipo sexual que se diferencien en cuanto a sus factores de virulencia, considerados estos de manera individual, muestren la misma patogenicidad. Además, podría ocurrir que el fondo genético de cada cepa de A. fumigatus juegue algún papel en la determinación del efecto del tipo sexual sobre la virulencia, tal y como se ha demostrado en el caso de $C$. neoformans serotipo $\mathrm{D}^{36}$.

\section{Conclusiones y perspectivas}

El descubrimiento de la fase sexual de A. fumigatus constituye un hito en la historia de la Micología. Sin embargo, pese a la innegable importancia de los hallazgos realizados hasta el momento, todavía quedan algunas cuestiones básicas por responder. ¿Muestran todas las cepas de este patógeno oportunista la misma capacidad para aparearse y completar su ciclo sexual? ¿Existe más de una fase teleomorfa? ¿Qué condiciones ambientales favorecen el apareamiento entre cepas compatibles y la producción de ascosporas? ¿Pueden las ascosporas de este hongo actuar como partículas infectivas? ¿Puede tener lugar la reproducción sexual de A. fumigatus dentro de un hospedador? Etcétera.

En conclusión, el estudio de la posible relación entre la reproducción sexual y la virulencia, tanto en el caso de A. fumigatus como de otros patógenos fúngicos, se postula como uno de los campos que va a centrar el interés de los micólogos durante los próximos años, no solo por su interés básico, sino también por las posibles repercusiones sobre el diagnóstico, la epidemiología y la terapéutica de las enfermedades que estos agentes ocasionan.

\section{Conflicto de intereses}

Los autores declaran no tener ningún conflicto de intereses.

\section{Agradecimientos}

Sergio Álvarez-Pérez es beneficiario de una beca predoctoral del Programa FPU del Ministerio de Educación y Ciencia (Ref. AP2005-1034). Los autores agradecen las sugerencias realizadas por dos revisores anónimos, las cuales sirvieron para centrar el enfoque de este artículo de revisión.

\section{Bibliografía}

1. Álvarez-Pérez S, Blanco JL, Alba P, García ME. Mating type and invasiveness are significantly associated in Aspergillus fumigatus. Med Mycol. 2009 doi:10.1080/ 13693780903095414.

2. Alvarez-Pérez S, García ME, Bouza E, Peláez T, Blanco JL. Characterization of multiple isolates of Aspergillus fumigatus from patients: genotype, mating type and invasiveness. Med Mycol. 2009;47:601-8.

3. Ayala FJ. Is sex better? Parasites say "no". Proc Natl Acad Sci USA. 1998;95: 3346-8.

4. Bain JM, Tavanti A, Davidson AD, Jacobsen MD, Shaw D, Gow NA, Odds FC Multilocus sequence typing of the pathogenic fungus Aspergillus fumigatus. J Clin Microbiol. 2007;45:1469-77.

5. Barton NH, Charlesworth B. Why sex and recombination? Science. 1998;281: 1986-90.

6. Bernstein C, Johns V. Sexual reproduction as a response to $\mathrm{H}_{2} \mathrm{O}_{2}$ damage in Schizosaccharomyces pombe. J Bacteriol. 1989;171:1893-7.

7. Blanco JL, Guedeja-Marrón J, Caballero J, García ME. Aspergilosis: mecanismos de patogenicidad implicados y aproximación al diagnóstico de laboratorio. Rev Iberoam Micol. 1998;15:10-5.

8. Blanco JL, Hontecillas R, Bouza E, Blanco I, Pelaez T, Muñoz P, Perez Molina J, Garcia ME. Correlation between the elastase activity index and invasiveness of clinical isolates of Aspergillus fumigatus. J Clin Microbiol. 2002;40: 1811-3.

9. Bouchara JP, Tronchin G, Larcher G, Chabasse D. The search for virulence determinants in Aspergillus fumigatus. Trends Microbiol. 1995;3:327-30.

10. Conde-Ferráez L. El locus MAT (mating-type) de los ascomicetos: su evolución, estructura y regulación. Rev Iberoam Micol. 2007;24:95-9.

11. Debeaupuis JP, Sarfati J, Chazalet V, Latgé JP. Genetic diversity among clinical and environmental isolates of Aspergillus fumigatus. Infect Immun. 1997;65: 3080-5.

12. Dyer PS, Paoletti M. Reproduction in Aspergillus fumigatus: sexuality in a supposedly asexual species? Med Mycol. 2005;43(Suppl.1):S7-14.

13. Dyer PS, Paoletti M, Archer DB. Genomics reveals sexual secrets of Aspergillus. Microbiology. 2003;149:2301-3.

14. Fan W, Kraus PR, Boily MJ, Heitman J. Cryptococcus neoformans gene expression during murine macrophage infection. Eukaryot Cell. 2005;4:1420-33. 
15. Fraser JA, Heitman J. Sex, MAT, and the evolution of fungal virulence. En: Heitman J, Filler SG, Edwards JE, Mitchell AP, editores. Molecular principles of fungal pathogenesis. Washington DC: ASM Press; 2006. p. 13-33.

16. Galagan JE, Calvo SE, Cuomo C, Ma LJ, Wortman JR, Batzoglou S, et al. Sequencing of Aspergillus nidulans and comparative analysis with A. fumigatus and A. oryzae. Nature. 2005;438:1105-15.

17. García ME, Caballero J, Blanco I, Cruzado M, Costas E, Blanco JL. Changes in the elastase activity and colonization ability of Aspergillus fumigatus after successive inoculations in mice. Rev Iberoam Micol. 2006;23:221-3.

18. Geiser DM. Sexual structures in Aspergillus: morphology, importance and genomics. Med Mycol. 2009;47(Supl. 1):S21-6.

19. Geiser DM, Frisvad JC, Taylor JW. Evolutionary relationships in Aspergillus section Fumigati inferred from partial $\beta$-tubulin and hydrophobin DNA sequences. Mycologia. 1998;90:831-45.

20. Gow NA. Fungal genomics: forensic evidence of sexual activity. Curr Biol. 2005;15:R509-11.

21. Guarro J, Gené J, Stchigel AM. Developments in fungal taxonomy. Clin Microbiol Rev. 1999;12:454-500.

22. Hawksworth DL. Separate name for fungus's sexual stage may cause confusion. Nature. 2009;458:29.

23. Heitman J. Sexual reproduction and the evolution of microbial pathogens. Curr Biol. 2006;16:R711-25.

24. Hernández-Molina JM, García-Martos P. Aislamiento de teleomorfos de muestras clínicas. Rev Iberoam Micol. 1998;15:235-42.

25. Hsueh YP, Heitman J. Orchestration of sexual reproduction and virulence by the fungal mating-type locus. Curr Opin Microbiol. 2008;11:517-24.

26. Hurst L, Peck JR. Recent advances in understanding of the evolution and maintenance of sex. Trends Ecol Evol. 1996;11:46-52.

27. Index Fungorum. An online database currently coordinated and supported by CABI Bioscience, CBS and Landcare Research [base de datos en línea; consultada el 13-10-2009]: Disponible en: http://www.speciesfungorum.org.

28. Kwon-Chung KJ, Bennett JE. Distribution of $\alpha$ and a mating types of Cryptococcus neoformans among natural and clinical isolates. Am J Epidemiol. 1978;108:337-40.

29. Kwon-Chung KJ, Edman JC, Wickes BL. Genetic association of mating types and virulence in Cryptococcus neoformans. Infect Immun. 1992;60:602-5.

30. Latgé JP. Aspergillus fumigatus and aspergillosis. Clin Microbiol Rev. 1999;12: 310-50.
31. Latgé JP. The pathobiology of Aspergillus fumigatus. Trends Microbiol. 2001;9:382-9.

32. Madrenys N, De Vroey C, Raes-Wuytack C, Torres-Rodríguez JM. Identification of the perfect state of Cryptococcus neoformans from 195 clinical isolates including 84 from AIDS patients. Mycopathologia. 1993;123:65-8.

33. Michod RE, Bernstein H, Nedelcu AM. Adaptive value of sex in microbial pathogens. Infect Genet Evol. 2008;8:267-85.

34. Nielsen K, Cox GM, Litvintseva AP, Mylonakis E, Malliaris SD, Benjamin Jr DK, et al. Cryptococcus neoformans $\alpha$ strains preferentially disseminate to the central nervous system during coinfection. Infect Immun. 2005;73: 4922-33.

35. Nielsen K, Heitman J. Sex and virulence of human pathogenic fungi. Adv Genet. 2007;57:143-73.

36. Nielsen K, Marra RE, Hagen F, Boekhout T, Mitchell TG, Cox GM, et al. Interaction between genetic background and the mating-type locus in Cryptococcus neoformans virulence potential. Genetics. 2005;171:975-83.

37. O'Gorman CM, Fuller HT, Dyer PS. Discovery of a sexual cycle in the opportunistic fungal pathogen Aspergillus fumigatus. Nature. 2009;457:471-4.

38. Otto SP, Lenormand T. Resolving the paradox of sex and recombination. Nat Rev Genet. 2002;3:252-61.

39. Paoletti M, Rydholm C, Schwier EU, Anderson MJ, Szakacs G, Lutzoni F, et al. Evidence for sexuality in the opportunistic fungal pathogen Aspergillus fumigatus. Curr Biol. 2005;15:1242-8.

40. Pöggeler S. Genomic evidence for mating abilities in the asexual pathogen Aspergillus fumigatus. Curr Genet. 2002;42:153-60.

41. Rementeria A, López-Molina N, Ludwig A, Vivanco AB, Bikandi J, Pontón J, et al. Genes and molecules involved in Aspergillus fumigatus virulence. Rev Iberoam Micol. 2005;22:1-23.

42. Rydholm C, Szakacs G, Lutzoni F. Low genetic variation and no detectable population structure in Aspergillus fumigatus compared to closely related Neosartorya species. Eukaryot Cell. 2006;5:650-7.

43. Shenoy BD, Jeewon R, Hyde KD. Impact of DNA sequence-data on the taxonomy of anamorphic fungi. Fungal Divers. 2007;26:1-54.

44. Varga J. Mating type gene homologues in Aspergillus fumigatus. Microbiology. 2003;149:816-9.

45. Varga J, Vida Z, Tóth B, Debets F, Horie Y. Phylogenetic analysis of newly described Neosartorya species. Anton Leeuw Int JG. 2000;77:235-9.

46. $\mathrm{Xu} \mathrm{J}$. The prevalence and evolution of sex in microorganisms. Genome. 2004;47:775-80. 